\title{
Principles of antimicrobial stewardship for bacterial and fungal infections in ICU
}

\author{
Matteo Bassetti ${ }^{*} \mathbb{D}$, Marin H. Kollef ${ }^{2}$ and Garyphallia Poulakou ${ }^{3}$
}

(C) 2017 Springer-Verlag GmbH Germany and ESICM

\section{Appropriate use of antibiotics}

Selecting antimicrobial therapy for suspected infection in critically ill patients is an important decision-making process for intensivists. In this current age of multidrug-resistant organisms (MDROs), intensivists must balance the increased mortality associated with delaying therapy of microbiologically documented infections with the potential ecological damage caused by antimicrobial medications, including the selection and development of MDROs [1]. In patients with septic shock, even delays of few hours in the administration of appropriate antimicrobial therapy, defined as the antibiotic regimen to which the causative pathogen is sensitive, has been associated with increased mortality [2]. The same concept applies to other types of documented infections, such as pneumonia or bloodstream infections, where administration of an inappropriate initial antibiotic regimen is associated with an increased risk of death that is not mitigated by treatment escalation [1, 2]. Unfortunately, the "appropriateness" of initial antibiotic regimens is becoming increasingly difficult due to the rising levels of bacterial resistance.

Escalating rates of antibiotic resistance, including resistance to carbapenems and colistin, are becoming increasingly prevalent and significantly impact morbidity, mortality, and costs related to infection in hospitalized patients, especially in the intensive care unit (ICU). In Europe, the prevalence of carbapenemase-producing Enterobacteriaceae is increasing, in particular with the rapid spread of carbapenem-hydrolysing oxacillinase- 48 and New Delhi metallo-beta-lactamase-producing

\footnotetext{
*Correspondence: mattba@tin.it; matteo.bassetti@uniud.it

${ }^{1}$ Infectious Diseases Clinic, Department of Medicine, University of Udine and Azienda Sanitaria Universitaria Integrata, Piazzale Santa Maria della Misericordia 15, 33100 Udine, Italy

Full author information is available at the end of the article
}

Enterobacteriaceae [3]. Antimicrobial use is a key determinant in the selection of resistant bacteria, whereby increasing daily cumulative exposure, especially for carbapenems, increases the likelihood of intestinal carriage of MDROs [3]. The rapid evolution of antibiotic resistance impedes efforts to insure that initial appropriate antibiotic therapy is delivered to critically ill infected patients. The rising rates of antibiotic resistance have likely contributed to the increase in observed mortality attributed to antibiotic-resistant bacteria, despite the overall reduction in deaths ascribed to infectious diseases in the last century [4]. Acinetobacter baumannii and Pseudomonas aeruginosa represent two of the most important MDROs due to the extent of their antimicrobial resistance, intrinsic virulence, especially for Pseudomonas, and ability to establish themselves within hospital environments such as ICUs and to infect immune suppressed hosts $[1,5]$. Indeed, a recent European task force now recommends the empiric use of polymixin or colistin as part of the empiric antimicrobial regimen when infection with $A$. baumannii is suspected due to the high rates of carbapenem resistance in this pathogen [5]. An important contributor to the escalating rates of infection with these MDROs, especially in Europe and North America, has been population migration from the Middle East and other parts of Asia and Africa. Similarly, the movement of individuals across borders is also recognized as an important source of spread of other emerging infections such as Dengue fever, Zika virus, and Middle East respiratory syndrome coronavirus [6]. Reducing the unnecessary use of antibiotics is seen as a pivotal strategy for curtailing the further emergence and spread of MDROs [7]. Figure 1 provides a summary of the strategy of antimicrobial de-escalation

\section{Springer}


that can assist intensivists in balancing the desire to treat with an appropriate antibiotic regimen with the need to avoid unnecessary antibiotic exposure.

\section{Catheter-related infections}

There is accumulating recent evidence that rates of catheter-related infections (CRI) can be substantially reduced towards zero with the correct application of care bundles. As recently summarized in Intensive Care Medicine, the cornerstone of prevention relies on the correct skin preparation [8, 9]. Alcohol seems mandatory in antisepsis solutions due to its rapid activity; chlorhexidine gluconate ensures a prolonged bactericidal effect. There are actually some important certainties/recommendations on prevention of CRI like: (1) application of sterile $2 \%$ CHG-alcohol to disinfect the skin prior to insertion of a vascular catheter, (2) no need for cleaning the skin with soap or detergent before antiseptic application when it is not obviously dirty or contaminated, (3) rinsing with sterile water is not recommended after antiseptic application, (4) single-use vials of disinfectant solutions are preferable to multiple-use bottles, (5) daily evaluation of the necessity of retaining central line(s) and removal if they are clinically no longer necessary, (6) non-adherent, soiled, or moistened dressings should be promply replaced, (7) a subclavian site of insertion is preferable unless otherwise contraindicated, (8) full barrier precautions should be used at insertion, (9) hand hygiene with alcoholic solution is mandatory before every manipulation, and (10) every unit should adopt a written protocol for catheter insertion and maintenance. Certainly, there is room for improvement, with behavioral approaches requiring welldesigned multinational trials. However, mortality and CRI rates were not reduced in a prospective randomized controlled study from Brazil employing a behavioral approach for retention or withdrawal of central lines; the rather short evaluation period might have contributed to this. Nevertheless, the feedback from administrators and caregivers was positive, prompting universal adoption of the program after the experimental period of 6 months [10]. Transparent antimicrobial dressings, being significantly more efficacious in preventing CRBSIs when compared to the reference dressings and the use of sterile $2 \%$ CHG/70\% isopropyl alcohol for skin antisepsis represent new standards of care $[8,9,11]$.

Source control, recommended within $12 \mathrm{~h}$ from patient's admission, includes primarily actions taken in the process of care to control the foci of infection. It

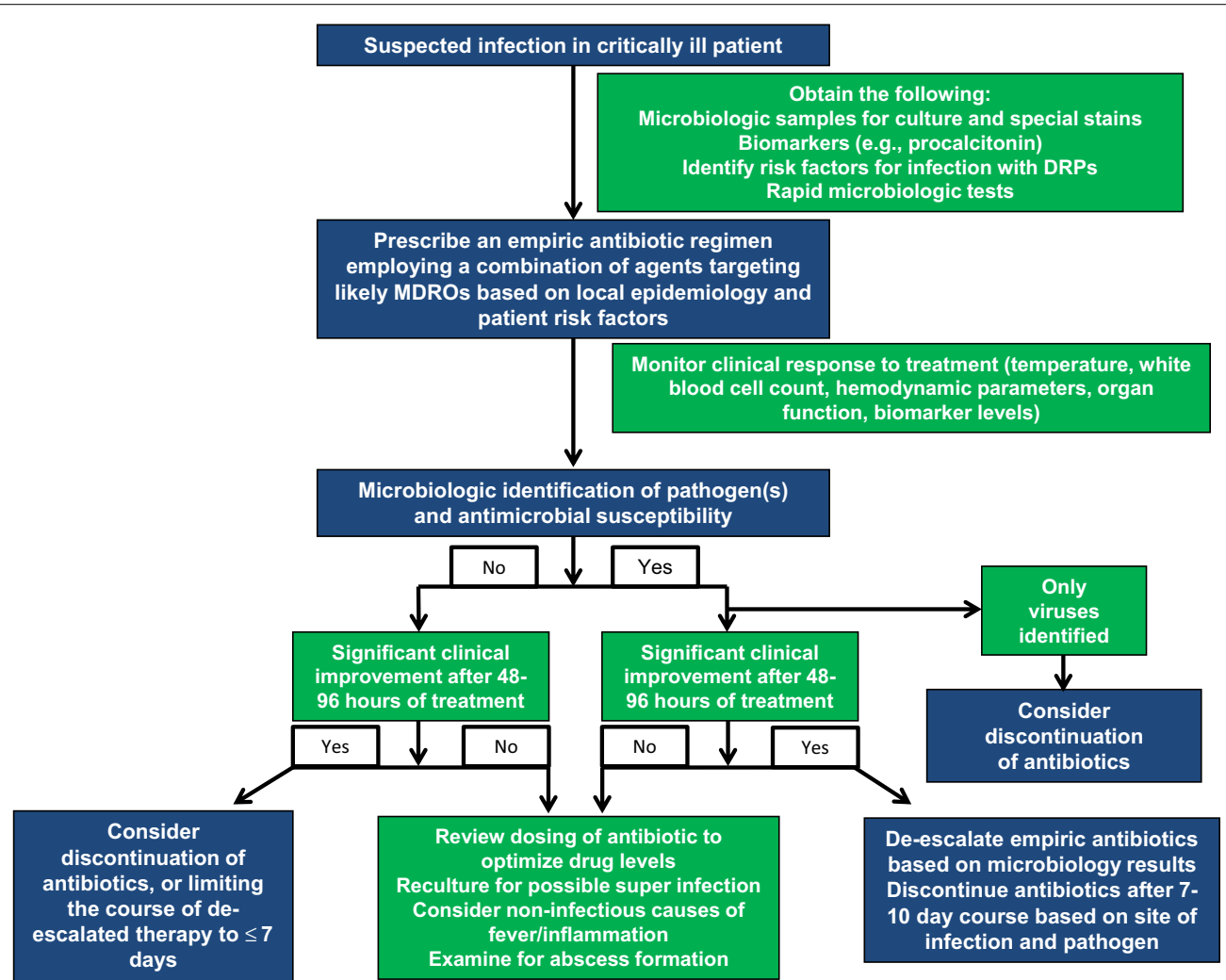

Fig. 1 Schematic outlining a practice of antimicrobial de-escalation. $\mathrm{FiO}_{2}$ inspired oxygen fraction, $\mathrm{PaO}_{2}$ partial pressure (or tension) of arterial oxygen, MDROs multi-drug resistant organisms 
is gaining importance as a pivotal measure to improve patients' outcomes, particularly in intra-abdominal, skin and soft tissue, implant-associated infections and CRI. In the Edusepsis study conducted in Spanish ICUs, patients who underwent source control were older, had a greater prevalence of shock, major organ dysfunction, bacteremia, inflammatory markers, lactic academia and worse compliance with the resuscitation bundle compared with those who did not undergo source control [12]. The source control group had lower crude ICU and hospital mortality even after adjustment for confounding factors. Source control performed after $12 \mathrm{~h}$ was not associated with higher mortality, implying that in high-risk patients even beyond the 12-h timeframe from admission, it may contribute to improved outcomes [5]. In another Spanish retrospective cohort of intraabdominal candidiasis (IAC), source control along with early antifungal treatment was associated with improved survival in both ICU and surgical wards, standing out as the main goal for decreasing mortality of IAC episodes inside and outside the ICU [13].

\section{Empiric and pre-emptive use of antifungals in Candida infections}

Invasive candidiasis (IC) comprises both bloodstream and other deep-seated invasive infections due to Candida species and represents a major cause of morbidity and mortality, which remains near $40 \%$ in most series. Over the past decades, incidence of IC has shown progressively increasing or stable rates in most regions [1416 ], probably due to the growing complexity of surgical procedures, the presence of patient populations at higher risk of infection, and the changes in patient demographic characteristics [14]. Attributable mortality around 10\% has been reported in several studies [16], while a recent well-designed study conducted in India has reported rates around $20 \%$ [14].

Infection-related morbidity and mortality associated with IC are in part associated with the critical illness itself, but they may also be attributed to suboptimal diagnostic tools. Standard mycological conventional blood culture is a gold standard, but may fail to diagnose IC in up to $25-50 \%$, whereas time to positivity for yeast and species identification can take several days from the time the blood culture is drawn, depending on the implicated Candida species [16]. Unless antifungal therapy is promptly initiated by the confirmation of IC, effective antifungal therapy may be further delayed. Numerous studies have demonstrated that the delay of initiation of antifungal therapy was associated with significant increases in both in-hospital mortality and the cost of care for patients with IC [16].
While candidemia episodes are easier to recognize and diagnose compared to deep-seated candidiasis, current guidelines mainly focus on the management of candidemia, while trials on IAC are lacking [15]. Risk factors for IAC include recent surgery, necrotizing pancreatitis, and anastomotic leaks. Empirical antifungal treatment with echinocandins or lipid-formulation of amphotericin B should be considered in the critically ill or in patients with previous exposure to azoles and risk factors for Candida spp. infection. Despite the lack of randomized trials, antifungal therapy for patients with complicated intra-abdominal infection is recommended when Candida spp. is grown from cultures [15].

In severe abdominal conditions in non-neutropenic critically ill patients, high 1,3--D-Glucan levels combined with Candida albicans germ tube antibodies may help clinicians in discriminating Candida spp. colonization from IC [17].

Source control has shown to be an important determinant of outcome for patients with candidemia and IAC [14-16]. Based on expert guidelines and previous studies, central venous catheter withdrawal should be attempted and any identified collection should be rapidly and appropriately drained.

In cases of IC, treatment should be given immediately, but thereafter it would be important to step down early to a triazole. De-escalation from an echinocandin to intravenous or oral fluconazole should be encouraged when the patient is clinically stable and the isolated strain is susceptible. The exact timing for shifting to fluconazole is not well established and may vary, depending on patient- and pathogen-related factors. The safety of deescalation (within 5 days) in the case of proven or probable IC has been recently suggested for non-neutropenic patients treated in the ICU, since it was not associated with increased mortality and led to a subsequent and significant decrease in the antifungal consumption [18].

The most remarkable rules for developing antifungal stewardship program targeting antifungal treatments bundles are reported in the Electronic Supplement Material.

\section{Electronic supplementary material \\ The online version of this article (doi:10.1007/s00134-017-4922-x) contains supplementary material, which is available to authorized users.}

\section{Abbreviations}

CRI: Catheter-related infections; IAC: Intraabdominal candidiasis; IC: Invasive candidiasis; ICU: Intensive care unit; MDROs: Multi-drug-resistant organisms.

\footnotetext{
Author details

${ }^{1}$ Infectious Diseases Clinic, Department of Medicine, University of Udine and Azienda Sanitaria Universitaria Integrata, Piazzale Santa Maria della Misericordia 15, 33100 Udine, Italy. ${ }^{2}$ Division of Pulmonary and Critical Care Medicine, Washington University School of Medicine, St. Louis, MO, USA. ${ }^{3}$ th Department of Internal Medicine, Attikon University General Hospital, Athens University School of Medicine, Athens, Greece.
} 


\section{Compliance with ethical standards}

\section{Conflicts of interest}

Dr. Bassetti has participated in advisory boards and/or received speaker honoraria from Achaogen, Angelini, Astellas, AstraZeneca, Bayer, Basilea, Gilead, Menarini, MSD, Pfizer, The Medicine Company, Tetraphase and Vifor. Dr. Kollef's effort was supported by the Barnes-Jewish Hospital Foundation. Dr Poulakou has participated in advisory boards and/or received speaker honoraria from MSD and Pfizer.

Received: 5 June 2017 Accepted: 14 June 2017

Published online: 12 September 2017

\section{References}

1. Bassetti M, De Waele JJ, Eggimann P, Garnacho-Montero J, Kahlmeter G, Menichetti F, Nicolau DP, Paiva JA, Tumbarello M, Welte T, Wilcox M, Zahar JR, Poulakou G (2015) Preventive and therapeutic strategies in critically ill patients with highly resistant bacteria. Intensive Care Med 41:776-795. doi:10.1007/s00134-015-3719-z

2. Liu VX, Fielding-Singh V, Greene JD, Baker JM, Iwashyna TJ, Bhattacharya J, Escobar GJ (2017) The timing of early antibiotics and hospital mortality in sepsis. Am J Respir Crit Care Med. doi:10.1164/rccm.201609-1848OC (Epub ahead of print)

3. Barbier F, Luyt CE (2016) Understanding resistance. Intensive Care Med 42:2080-2083. doi:10.1007/s00134-016-4543-9

4. Hansen V, Oren E, Dennis LK, Brown HE (2016) Infectious disease mortality trends in the United States, 1980-2014. JAMA 316:2149-2151. doi:10.1001/jama.2016.12423

5. Garnacho-Montero J, Dimopoulos G, Poulakou G, Akova M, Cisneros JM De Waele J, Petrosillo N, Seifert H, Timsit JF, Vila J, Zahar JR, Bassetti M, European Society of Intensive Care Medicine (2015) Task force on management and prevention of Acinetobacter baumannii infections in the ICU. Intensive Care Med 41:2057-2075. doi:10.1007/s00134-015-4079-4

6. Poulakou G, Plachouras D (2016) Planet's population on the move, infections on the rise. Intensive Care Med 42:2055-2058. doi:10.1007/ s00134-016-4580-4

7. Bretonnière C, Leone M, Milési C et al (2015) Strategies to reduce curative antibiotic therapy in intensive care units (adult and paediatric). Intensive Care Med 41:1181-1196. doi:10.1007/s00134-015-3853-7

8. Mimoz O, Chopra V, Timsit JF (2016) What's new in catheter-related infection: skin cleansing and skin antisepsis. Intensive Care Med 42:1784-1786

9. Mimoz O, Chopra V, Widmer A (2016) What's new in skin antisepsis for short-term intravascular catheters: new data to address old problems? Intensive Care Med 42:2043-2045

10. Mimoz O, Lucet JC, Kerforne T, Pascal J, Souweine B, Goudet V, Mercat A Bouadma L, Lasocki S, Alfandari S, Friggeri A, Wallet F, Allou N, Ruckly S, Balayn D, Lepape A, Timsit JF (2015) Skin antisepsis with chlorhexidinealcohol versus povidone iodine-alcohol, with and without skin scrubbing, for prevention of intravascular-catheterrelated infection (CLEAN): an open-label, multicentre, randomised, controlled, two-by-two factorial trial. Lancet 386:2069-2077
11. Writing Group for the CHECKLIST-ICU Investigators and the Brazilian Research in Intensive Care Network (BRICNet), Cavalcanti AB, Bozza FA, Machado FR, Salluh Jl, Campagnucci VP, Vendramim P, Guimaraes HP, Normilio-Silva K, Damiani LP, Romano E, Carrara F, Diniz Lubarino, de Souza J, Silva AR, Ramos GV, Teixeira C, Brandão da Silva N, Chang CC, Angus DC, Berwanger O (2016) Effect of a quality improvement intervention with daily round checklists, goal setting, and clinician prompting on mortality of critically ill patients: a randomized clinical trial. JAMA 315:1480-1490

12. Martínez ML, Ferrer R, Torrents E, Guillamat-Prats R, Gomà G, Suárez D, Álvarez-Rocha L, Pozo Laderas JC, Martín-Loeches I, Levy MM, Artigas A, Edusepsis Study Group (2017) Impact of source control in patients with severe sepsis and septic shock. Crit Care Med 45:11-19

13. Lagunes L, Rey-Pérez A, Martín-Gómez MT, Vena A, de Egea V, Muñoz P, Bouza E, Díaz-Martín A, Palacios-García I, Garnacho-Montero J, Campins M, Bassetti M, Rello J (2017) Association between source control and mortality in 258 patients with intra-abdominal candidiasis: a retrospective multi-centric analysis comparing intensive care versus surgical wards in Spain. Eur J Clin Microbiol Infect Dis 36:95-104. doi:10.1007/ s10096-016-2775-9

14. Chakrabarti A, Sood P, Rudramurthy SM, Chen S, Kaur H, Capoor M, Chhina D, Rao R, Eshwara VK, Xess I, Kindo AJ, Umabala P, Savio J, Patel A, Ray U, Mohan S, Iyer R, Chander J, Arora A, Sardana R, Roy I, Appalaraju B, Sharma A, Shetty A, Khanna N, Marak R, Biswas S, Das S, Harish BN, Joshi S, Mendiratta D (2015) Incidence, characteristics and outcome of ICUacquired candidemia in India. Intensive Care Med 41 (2):285-295

15. Bassetti M, Righi E, Ansaldi F, Merelli M, Scarparo C, Antonelli M, Garnacho-Montero J, Diaz-Martin A, Palacios-Garcia I, Luzzati R, Rosin C, Lagunes L, Rello J, Almirante B, Scotton PG, Baldin G, Dimopoulos G, Nucci M, Munoz P, Vena A, Bouza E, de Egea V, Colombo AL, Tascini C, Menichetti F, Tagliaferri E, Brugnaro P, Sanguinetti M, Mesini A, Sganga G, Viscoli C, Tumbarello M (2015) A multicenter multinational study of abdominal candidiasis: epidemiology, outcomes and predictors of mortality. Intensive Care Med 41(9):1601-1610. doi:10.1007/ s00134-015-3866-2

16. Bassetti M, Garnacho-Montero J, Calandra T, Kullberg B, Dimopoulos G, Azoulay E, Chakrabarti A, Kett D, Leon C, Ostrosky-Zeichner L, Sanguinetti M, Timsit JF, Richardson MD, Shorr A, Cornely OA (2017) Intensive care medicine research agenda on invasive fungal infection in critically ill patients. Intensive Care Med. doi:10.1007/s00134-017-4731-2

17. Martín-Mazuelos E, Loza A, Castro C, Macías D, Zakariya I, Saavedra P, RuizSantana S, Marín E, León C (2015) -D-Glucan and Candida albicans germ tube antibody in ICU patients with invasive candidiasis. Intensive Care Med 41(8):1424-1432

18. Bailly $\mathrm{S}$, Leroy $\mathrm{O}$, Montravers $\mathrm{P}$, Constantin JM, Dupont $\mathrm{H}$, Guillemot D, Lortholary O, Mira JP, Perrigault PF, Gangneux JP, Azoulay E, Timsit JF (2015) Antifungal de-escalation was not associated with adverse outcome in critically ill patients treated for invasive candidiasis: post hoc analyses of the AmarCAND2 study data. Intensive Care Med 41(11):1931-1940 